\title{
Approximations to the Length of an Arc.
}

\author{
By Professor D. M. Y. Sommerville.. \\ (Received 19th May 1919. Read 13th June 1919.)
}

§ 1. Dr E. M. Horsburgh* has given the approximate expression for the length of an arc of the catenary:

$$
s \rightarrow \frac{1}{3}(2 C+T)
$$

where $C$ is the length of the chord and $T$ the sum of the tangents at its extremities, analogous to Huygens' approximation to the length of a circular arc :

$$
s \rightarrow \frac{1}{3}\left(4 C^{\prime}-C\right)
$$

where $C^{\prime \prime}$ is the sum of the chords of the two halves of the arc. These approximations are not confined to these particular curves, but hold, within wider or narrower limits, for any continuous curve. In fact, if $s$ is regarded as a small quantity of the first order, as are also $C, C^{\prime}$ and $T$, the difference between $s$ and either of the quantities $\frac{1}{3}(2 C+T)$ and $\frac{1}{3}\left(4 C^{\prime}-C\right)$ is a small quantity of the fifth order. Other expressions of a similar form can be found involving the chords and tangents of the half-arcs.

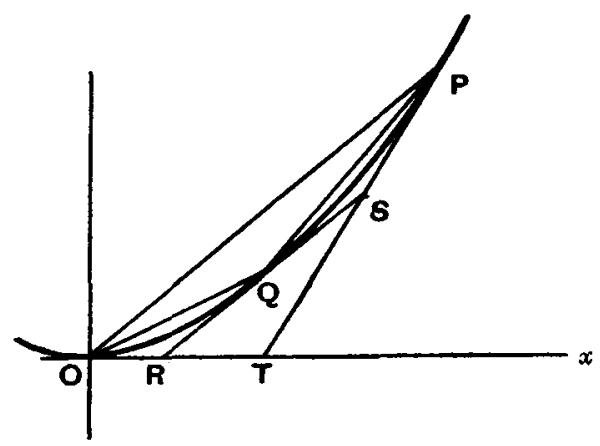

* Proc. Edinburgh Math. Soc., XXXVI. (1918), 94-95. 
§2. Consider any curve and a point $O$ upon it. Taking $O$ as origin and the tangent at $O$ as axis of $x$, the equation of the curve in the neighbourhood of $O$ can be written

$$
y=a_{0} x^{2}+a_{1} x^{2}+a_{2} x^{4}+\ldots
$$

We shail assume that $a_{0} \neq 0$, as otherwise the curve would have a point of inflexion at $O$.

To find $s$, we have

$$
d s=\left(1+p^{2}\right)^{\frac{1}{2}} d x
$$

where $p=d y / d x=2 a_{0} x+3 a_{1} x^{2}+4 a_{2} x^{3}+\ldots$,

whence $\left(1+p^{2}\right)^{\frac{1}{2}}=1+2 a_{0}^{2} x^{2}+6 a_{0} a_{1} x^{3}+\left(8 a_{0} a_{2}+\frac{9}{2} a_{1}^{2}-2 a_{0}^{4}\right) x^{4}+\ldots$.

Therefore $\quad 8=x+\frac{2}{3} a_{0}^{2} x^{3}+\frac{8}{2} a_{0} a_{1} x^{4}+\left(\frac{8}{5} a_{0} a_{2}+\frac{9}{10} a_{1}^{2}-\frac{2}{5} a_{0}^{4}\right) x^{5}+\ldots$.

Again $C^{2}=x^{2}+y^{2}$,

whence $C=x+\frac{1}{2} a_{0}^{2} x^{3}+a_{0} a_{1} x^{4}+\left(a_{0} a_{2}+\frac{1}{2} a_{1}^{2}-\frac{1}{8} a_{0}^{4}\right) x^{5}+\ldots$

Also the sum of the tangents $O T+T P$ is

$$
\begin{aligned}
T & =x+\left(\sqrt{1+p^{2}}-1\right) y / p \\
& =x+a_{0}^{2} x^{3}+\frac{5}{2} a_{0} a_{1} x^{4}+\left(3 a_{0} a_{2}+\frac{3}{2} a_{1}^{2}-a_{0}^{4}\right) x^{5}+\ldots .
\end{aligned}
$$

We have thus the approximations :

$$
\begin{aligned}
& s \rightarrow C+\frac{1}{6} a_{0}^{2} x^{3}+\frac{1}{2} a_{0} a_{1} x^{4}+\frac{1}{5}\left(3 a_{0} a_{2}+2 a_{1}^{8}-\frac{11}{8} a_{0}^{4}\right) x^{5}, \\
& s \rightarrow T-\frac{1}{3} a_{0}^{2} x^{3}-a_{0} a_{1} x^{4}-\frac{1}{6}\left(7 a_{0} a_{2}+3 a_{1}^{9}-3 a_{0}^{4}\right) x^{5},
\end{aligned}
$$

so that both $C$ and $T$ are second-order approximations to $s$.

Also we have, eliminating $x^{3}$,

$$
3 s \rightarrow 2 C+T-\frac{1}{5}\left(a_{0} a_{2}-a_{1}^{2}-\frac{1}{4} a_{0}^{4}\right) x^{5},
$$

so that $\frac{1}{3}(2 C+T)$ is a fourth-order approximation to 8 .

§3. Now divide the arc $O P$ at $Q$ so that the abseissa of $Q$ is $r x, r<1$, and let the sum of the chords $O Q+Q P=C^{\prime}$. Then we find that

$$
C^{\prime}=x+\frac{1}{2}\left(1+r-r^{2}\right) a_{0}^{2} x^{3}+\left(1+r-r^{3}\right) a_{0} a_{1} x^{4}+\ldots,
$$

and $s \rightarrow C^{\prime}+\frac{1}{6}\left(1-3 r+3 r^{2}\right) a_{0}^{2} x^{3}+\frac{1}{2}\left(1-2 r+2 r^{3}\right) a_{0} a_{1} x^{4}+\ldots$,

so that, for all values of $r, C^{\prime}$ is a second-order approximation to 8. A higher approximation, involving $C$ and $C^{\prime}$, analogous to that of Huygens, is then found by eliminating $x^{3}$ :

$$
3 r(1-r) s \rightarrow C^{\prime}-\left(1-3 r+3 r^{2}\right) C+\frac{1}{2} r(1-r)(1-2 r) a_{0} a_{1} x^{4} .
$$

The resulting expression is therefore only a third-order approximation for a general value of $r$, unless $a_{1}=0$. The latter condition holds if the curve is symmetrical about the normal, which, except 
for the circle (Huygens' case) can only happen at special points on the curve. If, however, $r=\frac{1}{2}$, we obtain, for any point on the curve, again a fourth-order approximation. In this case

$$
\begin{aligned}
C^{\prime} & =x+\frac{5}{8} a_{0}^{2} x^{3}+\frac{11}{8} a_{0} a_{1} x^{4}+\left(\frac{2 s}{18} a_{0} a_{2}+\frac{25}{32} a_{1}^{2}-\frac{41}{128} a_{0}^{4}\right) x^{5}+\ldots, \\
s & \rightarrow C^{\prime}+\frac{1}{24} a_{0}^{2} x^{3}+\frac{1}{8} a_{0} a_{1} x^{4}+\left(\frac{13}{80} a_{0} a_{2}+\frac{19}{180} a_{1}^{2}-\frac{51}{640} a_{0}^{4}\right) x^{*}, \\
\text { and } s & \rightarrow \frac{1}{3}\left(4 C^{\prime}-C\right)+\left(\frac{1}{80} a_{0} a_{2}+\frac{1}{40} a_{1}^{2}-\frac{7}{480} a_{0}^{4}\right) x^{3}
\end{aligned}
$$

If $r-\frac{1}{2}$ is of the same order of smallness as $x$, the expression $\frac{1}{3}\left(4 C^{\prime}-C\right)$ is still a fourth-order approximation.

\$4. Lastly, we shall take the sum of the tangents $T=O R+R S+S P$. The only difficulty is to find the sum $Q S+S P$, for which we obtain the expression

$\left\{\left(y_{2}-y_{1}\right)\left(\sqrt{1+p_{2}^{2}}-\sqrt{1+p_{1}^{2}}\right)+\left(x_{2}-x_{1}\right)\left(p_{2} \sqrt{1+p_{1}^{2}}-p_{1} \sqrt{1+p_{2}^{2}}\right)\right\} /\left(p_{2}-p_{1}\right)$, where $\left(x_{1} y_{1}\right),\left(x_{2} y_{2}\right)$ are the coordinates of $P, Q$, and $p_{1}, p_{2}$ are the values of $p$ at these points. Then we find

$$
T^{\prime}=x+\frac{3}{4} a_{0}^{2} x^{3}+\frac{7}{4} a_{0} a_{1} x^{4}+\left(\frac{31}{16} a_{0} a_{2}+\frac{9}{8} a_{1}^{2}-\frac{9}{16} a_{0}^{4}\right) x^{5}+\ldots
$$

Hence

$$
s \rightrightarrows T^{\prime}-\frac{1}{12} a_{0}^{2} x^{3}-\frac{1}{4} a_{0} a_{1} x^{4}-\left(\frac{27}{80} a_{0} a_{2}+\frac{9}{10} a_{1}^{2}-\frac{1}{80} a_{0}^{4}\right) x^{5},
$$

so that $T^{\prime}$ is also a second-order approximation.

In terms of the four second-order approximations, $C, T, C^{\prime}, T^{\prime}$, $s$ is represented thus

$$
\begin{aligned}
& C+\frac{1}{8} a_{0} x^{3}\left(a_{0}+3 a_{1} x\right)+\left(\frac{3}{5} a_{0} a_{2}+\frac{2}{5} a_{1}^{2}-\frac{11}{40} a_{0}^{3}\right) x^{5}, \\
& C^{\prime}+\frac{1}{24} a_{0} x^{3}\left(a_{0}+3 a_{1} x\right)+\left(\frac{23}{16} a_{0} a_{2}+\frac{25}{3} a_{1}^{2}-\frac{41}{128} a_{0}^{4}\right) x^{5}, \\
& T-\frac{1}{3} a_{0} x^{2}\left(a_{0}+3 a_{1} x\right)-\left(\frac{7}{5} a_{0} a_{2}+\frac{3}{5} a_{1}^{2}-\frac{3}{5} a_{0}^{4}\right) x^{5}, \\
& T^{\prime}-\frac{1}{12} a_{0} x^{3}\left(a_{0}+3 a_{1} x\right)-\left(\frac{27}{80} a_{0} a_{2}+\frac{9}{40} a_{1}^{2}-\frac{13}{80} a_{0}^{4}\right) x^{5} .
\end{aligned}
$$

$\$ 5$. By combining these in pairs we obtain the following six fourth-order approximations :

$$
\begin{aligned}
& \frac{1}{3}\left(4 C^{\prime}-C\right)+\frac{1}{60}\left(a_{0} a_{2}+\frac{3}{2} a_{1}^{2}-\frac{7}{8} a_{0}^{4}\right) x^{5} \\
& \frac{1}{3}(2 C+T)-\frac{1}{15}\left(a_{0} a_{2}-a_{1}^{2}-\frac{1}{4} a_{0}^{4}\right) x^{5}, \\
& \frac{1}{3}\left(C+2 T^{\prime}\right)-\frac{1}{40}\left(a_{0} a_{2}+\frac{2}{3} a_{1}^{2}-\frac{2}{3} a_{0}^{4}\right) x^{5} \\
& \frac{1}{8}\left(8 C^{\prime}+T\right)-\frac{1}{80}\left(a_{0} a_{2}-\frac{7}{2} a_{1}^{2}+\frac{3}{8} a_{0}^{4}\right) x^{5} \\
& \frac{1}{3}\left(2 C^{\prime}+T^{\prime}\right)-\frac{1}{240}\left(a_{0} a_{2}-a_{1}^{2}-\frac{1}{4} a_{0}^{4}\right) x^{5} \\
& \frac{1}{3}\left(4 T^{\prime}-T\right)+\frac{1}{60}\left(a_{0} a_{2}-6 a_{1}^{2}+a_{0}^{4}\right) x^{5}
\end{aligned}
$$


\$6. From the second and fifth we can eliminate $x^{5}$, and obtain a still higher approximation. It turns out that $x^{6}$ also goes, and we have the sixth-order approximation :

$$
\begin{aligned}
s \rightarrow & \frac{1}{65}\left(32 C^{\prime}+16 T^{\prime}-2 C-T\right) \\
& -\frac{1}{7}\left(\frac{1}{60} a_{0} a_{4}+\frac{1}{90} a_{1} a_{3}-\frac{1}{36} a_{2}^{2}-\frac{1}{15} a_{0}^{3} a_{2}-\frac{3}{80} a_{0}^{2} a_{1}^{2}+\frac{1}{96} a_{0}^{6}\right) x^{7} .
\end{aligned}
$$

Expansions of 8, $C, C^{\prime}, T, T^{\prime}$ up to $x^{7}:-$

$$
\begin{aligned}
8=x+\frac{2}{3} a_{0}^{2} & x^{3}+\frac{3}{2} a_{0} a_{1} x^{4}+\left(\frac{8}{5} a_{0} a_{2}+\frac{9}{10} a_{1}^{2}-\frac{2}{5} a_{0}^{4}\right) x^{5} \\
& +\left(\frac{5}{3} a_{0} a_{3}+2 a_{1} a_{2}-2 a_{0}^{3} a_{1}\right) x^{6} \\
& +\left(\frac{12}{7} a_{0} a_{4}+\frac{15}{7} a_{1} a_{3}+\frac{8}{7} a_{2}^{2}-\frac{16}{7} a_{0}^{3} a_{2}-\frac{27}{7} a_{0}^{2} a_{1}^{2}+\frac{4}{7} a_{0}^{6}\right) x^{7}
\end{aligned}
$$$$
C=x+\frac{1}{2} a_{0}^{2} x^{3}+a_{0} a_{1} x^{4}+\left(a_{0} a_{2}+\frac{1}{2} a_{1}^{2}-\frac{1}{8} a_{0}^{4}\right) x^{5}+\left(a_{0} a_{3}+a_{1} a_{2}-\frac{1}{2} a_{0}^{3} a_{1}\right) x^{6}
$$$$
+\left(a_{0} a_{4}+a_{1} a_{3}+\frac{1}{2} a_{2}^{2}-\frac{1}{2} a_{0}^{3} a_{2}-\frac{3}{4} a_{0}^{2} a_{1}^{2}+\frac{1}{16} a_{0}^{6}\right) x^{7} \text {. }
$$$$
C^{\prime}=x+\frac{5}{8} a_{0}^{2} x^{3}+\frac{11}{8} a_{0} a_{1} x^{4}+\left(\frac{23}{16} a_{0} a_{2}+\frac{35}{3} \frac{5}{2} a_{1}^{2}-\frac{41}{128} a_{0}^{4}\right) x^{5}
$$$$
+\left(\frac{47}{32} a_{0} a_{3}+\frac{53}{32} a_{1} a_{2}-\frac{95}{84} a_{0}^{3} a_{1}\right) x^{6}
$$$$
+\left(\frac{98}{\partial 4} a_{0} a_{4}+\frac{109}{64} a_{1} a_{3}+\frac{113}{128} a_{2}^{2}-\frac{208}{12 \frac{8}{8}} a_{0}^{3} a_{2}-\frac{683}{286} a_{0}^{2} a_{1}^{9}+\frac{385}{1024} a_{0}^{6}\right) x^{7} \text {. }
$$$$
T=x+a_{0}^{2} x^{3}+\frac{8}{2} a_{0} a_{1} x^{4}+\left(3 a_{0} a_{2}+\frac{3}{2} a_{1}^{2}-a_{0}^{4}\right) x^{5}
$$$$
+\left(\frac{7}{2} a_{0} a_{3}+\frac{7}{2} a_{1} a_{2}-\frac{11}{2} a_{0}^{3} a_{1}\right) x^{6}
$$$$
+\left(4 a_{0} a_{4}+4 a_{1} a_{3}+2 a_{2}^{2}-7 a_{0}^{3} a_{2}-\frac{45}{4} a_{0}^{2} a_{1}^{2}+2 a_{0}^{6}\right) x^{7}
$$$$
T^{\prime}=x+\frac{3}{4} a_{0}^{2} x^{3}+\frac{7}{4} a_{1} a_{1} x^{4}+\left(\frac{31}{16} a_{0} a_{2}+\frac{9}{8} a_{1}^{2}-\frac{9}{16} a_{0}^{4}\right) x^{5}
$$$$
+\left(\frac{67}{32} a_{0} a_{3}+\frac{85}{32} a_{1} a_{2}-\frac{49}{16} a_{0}^{3} a_{1}\right) x^{8}
$$$$
+\left(\frac{143}{84} a_{0} a_{4}+3 a_{1} a_{3}+\frac{13}{8} a_{2}^{2}-\frac{241}{64} a_{0}^{3} a_{2}-\frac{1659}{256} a_{0}^{2} a_{1}^{2}+\frac{38}{32} a_{0}^{6}\right) x^{7} \text {. }
$$

$$
\begin{aligned}
Q S+ & S P=\frac{1}{2} x+\frac{5}{8} a_{0}^{2} x^{3}+\frac{51}{32} a_{0} a_{1} x^{4}+\left(\frac{59}{3} \frac{9}{2} a_{0} a_{2}+\frac{89}{64} a_{1}^{2}-\frac{17}{32} a_{0}^{4}\right) x^{5} \\
& +\left(\frac{281}{128} a_{0} a_{3}+\frac{333}{128} a_{1} a_{2}-\frac{381}{12} \frac{1}{8} a_{0}^{3} a_{1}\right) x^{6} \\
& +\left(\frac{141}{84} a_{0} a_{4}+\frac{95}{32} a_{1} a_{3}+\frac{103}{64} a_{2}^{2}-\frac{475}{12} \frac{7}{28} a_{0}^{3} a_{2}-\frac{3273}{512} a_{0}^{2} a_{1}^{2}+\frac{85}{64} a_{0}^{6}\right) x^{7}
\end{aligned}
$$

